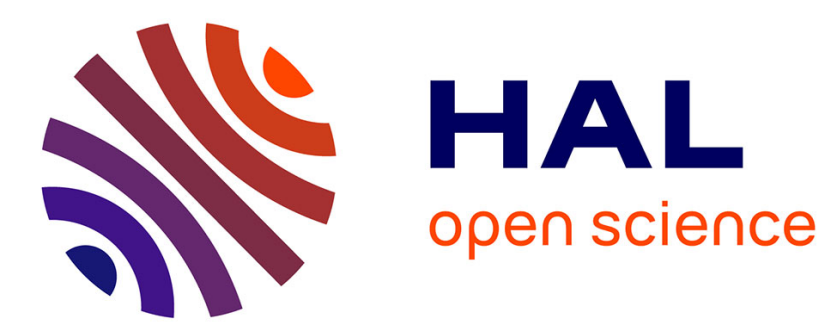

\title{
Parametric and nonparametric models of the impedance matrix of a random medium
}

R. Cottereau, Didier Clouteau, Christian Soize

\section{To cite this version:}

R. Cottereau, Didier Clouteau, Christian Soize. Parametric and nonparametric models of the impedance matrix of a random medium. Revue Européenne de Mécanique Numérique/European Journal of Computational Mechanics, 2008, 17 (5-7), pp.881-892. hal-00686299

\section{HAL Id: hal-00686299 \\ https://hal.science/hal-00686299}

Submitted on 9 Apr 2012

HAL is a multi-disciplinary open access archive for the deposit and dissemination of scientific research documents, whether they are published or not. The documents may come from teaching and research institutions in France or abroad, or from public or private research centers.
L'archive ouverte pluridisciplinaire HAL, est destinée au dépôt et à la diffusion de documents scientifiques de niveau recherche, publiés ou non, émanant des établissements d'enseignement et de recherche français ou étrangers, des laboratoires publics ou privés. 


\title{
Parametric and nonparametric models of the impedance matrix of a random medium
}

\author{
Régis Cottereau* - Didier Clouteau* - Christian Soize** \\ * École Centrale Paris, Laboratoire MSSMat, \\ Grande Voie des Vignes, 92295 Châtenay-Malabry, France \\ \{regis.cottereau,didier.clouteau\}@ecp.fr \\ ** Université Paris-Est, Laboratoire de Mécanique, \\ 5 bd Descartes, 77454 Marne-la-Vallée, France \\ soize@univ-mlv.fr
}

\begin{abstract}
Two approaches are presented for the modeling of the impedance matrix of a random medium: one parametric and the other nonparametric. The former allows to take into account the data uncertainties while introducing a model error, that yields, in some cases, very high levels. The latter is based on a much simpler, deterministic, model, for which both data uncertainties and model errors are accounted for. When the model error is negligible, the parametric approach can be used for the identification of the parameters of the nonparametric model of the impedance matrix.

RÉSUMÉ. Deux approches sont présentées pour la modélisation de la matrice d'impédance d'un milieu aléatoire: une paramétrique et une non-paramétrique. La première prend en compte les erreurs de données, mais introduit une erreur de modèle, qui peut, selon les cas, atteindre des niveaux importants. La seconde est basée sur un modèle simpliste et déterministe, pour lequel à la fois les erreurs de données et de modèle peuvent être prises en compte. Lorsque l'erreur de modèle est négligeable, l'approche paramétrique peut être utilisée pour l'identification des paramètres du modèle non-paramétrique de la matrice d'impédance.
\end{abstract}

KEYWORDS: Probabilistic methods, Impedance matrix, Unbounded domain.

MOTS-CLÉS : Méthodes probabilistes, Matrice d'impédance, Domaine non-borné.

$1^{\text {re }}$ soumission à European Journal of Computational Mechanics, le September 3, 2007 


\section{Introduction}

In many fields of application, as civil engineering or aeronautics, engineers have to design structures that are in contact with unbounded domains. In these applications, only the structure is really of interest for the engineers, and the exterior domain is important only through its equivalent stiffness, in statics, or its boundary impedance matrix, in dynamics.

The unbounded domains that are considered in these applications are often inaccurately described and complex. For instance, the natural heterogeneity of a soil is often replaced, in seismic design problems, by a system of homogeneous horizontal layers. However, the physical behavior of such a simplified system is quite different from the original, and some features are then lost. The error that is introduced is referred to as model error. Besides, once the model has been chosen, the shear size of the domains that are considered hinders the feasibility of an experimental campaign to assess the parameters of that model. The uncertainty associated with the evaluation of these parameters is referred to as data uncertainty. For some domains, and in particular the soil in geotechnical problems, these data and model uncertainties can be very large. Probabilistic approaches can then be introduced to try and take them into account.

Two types of approaches are possible: a parametric, which takes into account data uncertainties only, and a nonparametric, which can assess both model errors and data uncertainties. The former consists in constructing probabilistic models of the parameters of the mechanical system, and to deduce the corresponding stochastic model for the impedance matrix. The most widely used method for this approach is the Stochastic Finite Element (SFE) method (Ghanem et al., 1991). The nonparametric approach consists in taking into account uncertainties directly at the level of the matrices of the considered dynamical system. Based on the original method by Soize (Soize, 2000; Soize, 2001), presenting the construction of a probabilistic model for the generalized matrices of mass, damping and stiffness of a dynamical system, a nonparametric probabilistic model for impedance matrices was recently introduced (Cottereau, 2007; Cottereau et al., 2007a; Cottereau et al., 2007b).

We propose here to construct, for a common reference problem ( $c f$. section 2), probabilistic models of the boundary impedance matrix, following successively a parametric approach ( $c f$. section 3.1) and a nonparametric approach ( $c f$. section 3.2). When the model error that is introduced in the parametric approach is negligible, this method can be used for the identification of the parameters of the nonparametric model of the impedance matrix ( $c f$. section 3.3). An example is provided, of a rigid embedded foundation within a layer of random soil on a rigid bedrock ( $c f$. section 4 ).

\section{Reference problem}

Let us consider a general domain $\Omega$, which is considered random in the sense that its mechanical properties are modeled as random fields. This domain $\Omega$ may be unbounded, and its boundary is denoted $\partial \Omega$. We define part of this boundary as a 


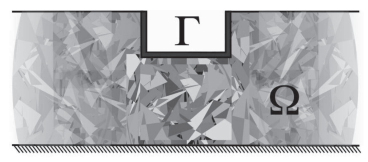

(a) Reference model

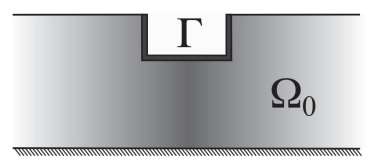

(b) Mean model

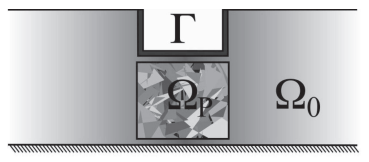

(c) SFE model

Figure 1. (a) Reference problem of a rigid embedded foundation in a horizontallyunbounded layer of random soil on top of a rigid bedrock, (b) corresponding mean model, and (c) SFE model of the reference problem.

boundary of interest $\Gamma$, with respect to which we want to define the impedance matrix. For instance, this boundary $\Gamma$ would be the interface between the soil and the structure in seismic design of a structure (see figure 1(a)), or the union of the interfaces between the soil and the structure and between the fluid and the structure in dam engineering. Free field conditions are enforced on part of the boundary, denoted $\partial \Omega_{\sigma}$, and clamped conditions are imposed on another part, denoted $\partial \Omega_{u}$. We suppose that $\Gamma \cap \partial \Omega_{\sigma}=\emptyset$, $\Gamma \cap \partial \Omega_{u}=\emptyset, \partial \Omega_{u} \cap \partial \Omega_{\sigma}=\emptyset$, and $\Gamma \cup \partial \Omega_{\sigma} \cup \partial \Omega_{u}=\partial \Omega$.

The Lamé's parameters $\boldsymbol{\lambda}(\mathbf{x})$ and $\boldsymbol{\mu}(\mathbf{x})$ of the random medium $\Omega$ are modeled as the restrictions on $\Omega$ of second-order homogeneous random fields, defined on a probabilistic space $(\mathcal{A}, \mathcal{T}, \mathrm{P})$, and indexed on $\mathbb{R}^{3}$. The corresponding mean fields are constant and denoted by

$$
\lambda_{0}=\mathrm{E}[\boldsymbol{\lambda}(\mathbf{x})], \mu_{0}=\mathrm{E}[\boldsymbol{\mu}(\mathbf{x})],
$$

and the covariance is such that

$$
C_{\alpha \beta}\left(\mathbf{x}, \mathbf{x}^{\prime}\right)=C_{\alpha \beta}\left(\left|\mathbf{x}-\mathbf{x}^{\prime}\right|\right)=\mathrm{E}\left[\left(\boldsymbol{\alpha}(\mathbf{x})-\alpha_{0}\right)\left(\boldsymbol{\beta}\left(\mathbf{x}^{\prime}\right)-\beta_{0}\right)\right],
$$

where $\boldsymbol{\alpha}$ and $\boldsymbol{\beta}$ stand either for $\boldsymbol{\lambda}$ or $\boldsymbol{\mu}$, and $\alpha$ and $\beta$ for $\lambda$ or $\mu$. For simplicity, the unit mass $\rho(\mathbf{x})$ of the medium will be supposed constant throughout this paper.

The harmonic boundary value problem (BVP) in $\Omega$ that is used to define the impedance matrix consists in finding, for each $\omega \in \mathbb{R}$, a displacement field $\mathbf{u}=$ $\left[\mathrm{u}_{\mathrm{i}}\right]_{1 \leq \mathrm{i} \leq 3}$ such that, for $1 \leq i \leq 3$,

$$
\begin{cases}\sigma_{i j, j}(\mathbf{u})+\rho(\mathbf{x}) \omega^{2} \mathrm{u}_{\mathrm{i}}=0 & \text { in } \Omega \\ \mathrm{u}_{\mathrm{i}}=\phi_{\mathrm{i}} & \text { on } \Gamma \\ \sigma_{i j}(\mathbf{u}) \mathrm{n}_{\mathrm{j}}=0 & \text { on } \partial \Omega_{\sigma}, \\ \mathrm{u}_{\mathrm{i}}=0 & \text { on } \partial \Omega_{u}\end{cases}
$$

where $\phi=\left[\phi_{i}\right]_{1 \leq i \leq 3}$ is a given displacement field imposed on the boundary $\Gamma, \mathbf{n}=$ $\left[\mathrm{n}_{\mathrm{i}}\right]_{1 \leq \mathrm{i} \leq 3}$ is the normal to boundary $\partial \Omega_{\sigma}$, and $\sigma(\mathbf{u})$ is the elastic stress tensor,

$$
\sigma_{i j}(\mathbf{u})=\boldsymbol{\lambda}(\mathbf{x}) \mathrm{u}_{\mathrm{k}, \mathrm{k}} \delta_{\mathrm{ij}}+\boldsymbol{\mu}(\mathbf{x})\left(\mathrm{u}_{\mathrm{j}, \mathrm{i}}+\mathrm{u}_{\mathrm{i}, \mathrm{j}}\right) .
$$


Implicit summation over repeated indexes is considered, and an index following a comma indicates a derivation with respect to the corresponding coordinate. $\delta_{i j}$ represents Kronecker's delta.

The solution $\mathbf{u}(\mathbf{x})$ of the BVP of eq. [3] corresponds to a traction field $\mathbf{f}_{\Gamma}$ on $\Gamma$. By definition, the impedance operator relates $\mathbf{f}_{\Gamma}$ to the imposed displacement field $\phi$ on $\Gamma$. When these two fields are approximated by their expansions on a common finite Hilbert basis of functions indexed on $\Gamma$, the boundary impedance matrix $\left[\mathbf{Z}_{s}(\omega)\right]$ is defined, with respect to the coordinates $\left[\mathbf{f}_{\Gamma}(\omega)\right]$ and $[\Phi(\omega)]$ of these expansions, by

$$
\left[\mathbf{Z}_{s}(\omega)\right][\Phi(\omega)]=\left[\mathbf{f}_{\Gamma}(\omega)\right] .
$$

The problem that was described in this section, where the Lamé's parameters are modeled as random fields, is referred to as the "reference problem" (figure 1(a)). It yields the definition of the "reference impedance matrix", $\left[\mathbf{Z}_{s}(\omega)\right]$.

\section{Probabilistic models of the impedance matrix}

In the general case, it is not possible to compute $\left[\mathbf{Z}_{s}(\omega)\right]$. Therefore, two approaches are introduced for the modeling of the impedance matrix of the reference problem of figure 1(a): a parametric one (figure 1(c)), and a nonparametric one. The former consists in considering $\boldsymbol{\lambda}(\mathbf{x})$ and $\boldsymbol{\mu}(\mathbf{x})$ in the BVP [3] as random only on part of the random domain $\Omega$, and constant elsewhere, at their mean value $\lambda_{0}$ and $\mu_{0}$. Besides possible data uncertainties, this process induces a model error that cannot be accounted for. On the other hand, the nonparametric model is based on a "mean" model (figure 1(b)), where the Lamé's parameters are taken as constant everywhere, therefore inducing a large model error. However, in this approach, both data uncertainties and model error can be taken into account.

\subsection{Parametric model of the impedance matrix $\left[\mathrm{Z}_{P}(\omega)\right]$}

In this approach, we subdivide $\Omega$ into a bounded part $\Omega_{P}$, where the Lamé's parameters will be modeled by random fields, as in the "reference problem", and its complement $\Omega_{0}$, where they will be taken as constant, at values $\lambda_{0}$ and $\mu_{0}$. This is typically what happens when the SFE method is used to solve a problem where random fields are defined over an unbounded domain. Indeed, this method requires the random fields to be discretized over a FE grid, which means that unbounded domains must be cut out in some fashion.

A possible approach, extending the classical, deterministic, FE modeling of unbounded domains, would consist in introducing absorbing boundary layers, or absorbing boundary conditions at some distance from the excitation and observation points (Magoulès et al., 2006). However, no generalization seem to exist yet for problems with random distributions of the mechanical parameters. Another approach (Savin et al., 2002), that will be used here, consists in coupling the bounded 
domain $\Omega_{P}$, that will be modeled by the SFE method, to the unbounded deterministic domain $\Omega_{0}$, that can be modeled, for example, by the Boundary Element (BE) method.

Within the bounded domain $\Omega_{P}$, the covariance function (eq. [2]) of the Lamés parameters is supposed to be known. The fields of parameters can then be written as Karhunen-Loève expansions

$$
\boldsymbol{\lambda}(\mathbf{x})=\lambda_{0}+\sum_{\ell=1}^{\infty} \boldsymbol{\xi}_{\ell} e_{\ell}(\mathbf{x}), \boldsymbol{\mu}(\mathbf{x})=\mu_{0}+\sum_{\ell=1}^{\infty} \boldsymbol{\kappa}_{\ell} e_{\ell}(\mathbf{x})
$$

where the $\left\{\boldsymbol{\xi}_{\ell}\right\}_{\ell \geq 1}$ and $\left\{\boldsymbol{\kappa}_{\ell}\right\}_{\ell \geq 1}$ are uncorrelated random variables, and the $\left\{e_{\ell}\right\}_{\ell \geq 1}$ are eigenfunctions of the covariance operator in eq. [2]. In practice, the sums in eq. [6] are truncated after $N_{K H}$ modes, which is chosen such that the trace of the covariance operator is well represented.

We additionally suppose that realizations of the $\boldsymbol{\lambda}(\mathbf{x})$ and $\boldsymbol{\mu}(\mathbf{x})$ can be constructed by drawing independent realizations of the $\left\{\boldsymbol{\xi}_{\ell}\right\}_{\ell \geq 1}$ and $\left\{\boldsymbol{\kappa}_{\ell}\right\}_{\ell \geq 1}$ with an uniform random variables generator, and discarding those realizations of the $\boldsymbol{\lambda}(\mathrm{x})$ and $\boldsymbol{\mu}(\mathrm{x})$ that reach a negative value for some $\mathbf{x}$. For each acceptable realization of the Lamé's parameters fields, a realization of the soil impedance matrix can be computed, by solving the BVP [3]. The probability law of $\left[\mathbf{Z}_{\mathrm{P}}(\omega)\right]$ can then be estimated through statistics of these realizations.

\subsection{Nonparametric model of the impedance matrix $\left[\mathrm{Z}_{N P}(\omega)\right]$}

The nonparametric approach is quite different. The reference problem, where the mechanical fields are random is replaced by a very simplistic one, the mean model, where they are constants. This mean model of the impedance matrix $\left[\mathrm{Z}_{0}(\omega)\right]$ therefore includes both data and model error, with respect to $\left[\mathbf{Z}_{s}(\omega)\right]$. The nonparametric model of the impedance matrix $\left[\mathbf{Z}_{\mathrm{NP}}(\omega)\right]$ is therefore introduced, based on the mean model, to take these errors into account.

The nonparametric method was originally introduced in structural vibration problems for the modeling of matrices of mass, damping and stiffness (Soize, 2000; Soize, 2001), and recently extended to impedance matrices (Cottereau et al., 2007a; Cottereau, 2007; Cottereau et al., 2007b), through the use of so-called "hidden variables models". In this approach, the probabilistic model of the considered matrix is constructed, around a known mean model, by enforcing that each realization of the matrix verify a given set of algebraic conditions. These conditions, for the impedance matrix, include that it be causal and stable.

The parameters that have to be identified for the construction of the nonparametric model of the impedance matrix are the mean model $\left[\mathrm{Z}_{0}(\omega)\right]$, and a set of dispersion parameters, denoted $\delta_{\mathrm{K}}, \delta_{\mathrm{D}}$, and $\delta_{\mathrm{M}}$. These parameters control the scattering, around their mean value, of the realizations of the matrices of stiffness $[\mathbf{K}]$, damping $[\mathbf{D}]$, and mass $[\mathbf{M}]$, of the hidden variables model of the impedance matrix. The choice of these 
parameters is usually made by comparison with experiments (Soize, 2005; Arnst et al., 2007; Arnst, 2007). In the next section, we discuss the possibility to identify them by comparison with numerical experiments constructed with the parametric approach.

The practical construction of $\left[\mathbf{Z}_{\mathrm{NP}}(\omega)\right]$ is done in the following way:

1) Computation of $\left[Z_{0}(\omega)\right]$ using any method.

2) Identification of the matrices of stiffness $\left[K_{0}\right]$, damping $\left[D_{0}\right]$, and mass $\left[M_{0}\right]$, of the hidden variables model of $\left[\mathrm{Z}_{0}(\omega)\right]$, as described in (Cottereau et al., 2007a).

3) Computation of realizations of the nonparametric probabilistic models of $[\mathbf{K}]$, [D], and [M], as described, for example, in (Soize, 2001).

4) Computation, by condensation, of realizations of $\left[\mathbf{Z}_{\mathrm{NP}}(\omega)\right]$.

5) Estimation of statistics of $\left[\mathbf{Z}_{\mathrm{NP}}(\omega)\right]$.

\subsection{Identification of the parameters of the nonparametric model $\left[\mathrm{Z}_{N P}(\omega)\right]$}

In the previous two sections, two probabilistic models of the impedance matrix have been constructed in two very different ways. The first one is constructed by the propagation of randomness on the parameters of the model towards the impedance matrix, and the other is built directly at the level of the impedance matrix, by enforcing a given set of algebraic conditions. The models of the medium on which they are built are also very different: one is a random medium, although partially, while the other is deterministic. Finally, they do not span the same spaces of matrix-valued random processes. However, they aim at representing the same reference problem.

In both cases, model errors are introduced during the modeling process. In the nonparametric approach these model errors can be accounted for, while they cannot be dealt with in the parametric approach. If it is possible to construct a parametric model of the impedance matrix $\left[\mathbf{Z}_{\mathrm{P}}(\omega)\right]$ in which the model error is negligible, then a numerical experiment can be conducted that generates realizations of the impedance matrix of the reference medium, and accounting for data uncertainties. The parameters of the nonparametric model of the impedance matrix $\left[\mathbf{Z}_{\mathrm{NP}}(\omega)\right]$, assessing both data and model errors, can then be identified from the results of this numerical experiment.

In the next section, we will construct a parametric model of the impedance matrix $\left[\mathbf{Z}_{\mathrm{P}}(\omega)\right]$ for which the model error is important for some elements and negligible for others (in particular the shaking element). In that case, the parameters of the nonparametric model $\left[\mathbf{Z}_{\mathrm{NP}}(\omega)\right]$ should be identified only on the latter elements.

\section{Impedance matrix of a random layer of soil on top of a rigid bedrock}

In this section, we apply the principles seen in the previous sections, to a particular example of the impedance matrix of a rigid embedded foundation on a random layer of soil, over a rigid bedrock. 


\subsection{Reference model of the impedance matrix}

The geometry of the reference problem is that presented in figure 1(a), with the height of layer $H$, three times larger than the radius of the foundation $R$, and the height of the embedment $D$. The foundation is rigid and circular.

$$
H=3 R=3 D=30 \mathrm{~m}
$$

The unit mass is constant at $\rho_{0}=2000 \mathrm{~kg} / \mathrm{m}^{3}$, and the mean Lamé's parameters are $\lambda_{0}=0.36 \mathrm{GPa}$ and $\mu_{0}=0.18 \mathrm{GPa}$, corresponding to mean compressional and shear velocities $v_{P, 0}=600 \mathrm{~m} / \mathrm{s}$ and $v_{S, 0}=300 \mathrm{~m} / \mathrm{s}$.

\subsection{Parametric model of the impedance matrix}

Let us first construct the parametric model of the soil impedance matrix $\left[\mathbf{Z}_{\mathrm{P}}(\omega)\right]$. We suppose that the correlation structure of the Lamé's parameters is of the exponential type, with an isotropic correlation length $L_{c}$,

$$
C_{\alpha \beta}\left(\left|\mathbf{x}-\mathbf{x}^{\prime}\right|\right)=c_{\alpha} c_{\beta} \exp \left(-\frac{\left|\mathbf{x}-\mathbf{x}^{\prime}\right|}{L_{c}}\right),
$$

where $\alpha$ and $\beta$ are either $\lambda$ or $\left.\mu, c_{\lambda}=\sqrt{(} C_{\lambda \lambda}\right)$, and $\left.c_{\mu}=\sqrt{(} C_{\mu \mu}\right)$. We consider $c_{\lambda}=\gamma \lambda_{0}$ and $c_{\mu}=\gamma \mu_{0}$, with $\gamma=60 \%$.

Three different correlation lengths are considered: $L_{c}=5 \mathrm{~m}, L_{c}=10 \mathrm{~m}$, and $L_{c}=20 \mathrm{~m}$. The Karhunen-Loève expansion (eq. [6]) is truncated after $N_{K H}=20$ modes, which corresponds to an error in the evaluation of the trace of the covariance matrix of around $15 \%$ in the three cases that were considered. 1000 Monte Carlo trials are drawn in each case.

The real part of the parametric model of two elements of the soil impedance matrix are drawn in figure 2, in the case of $L_{c}=10 \mathrm{~m}$. In figure 3, a frequency-wise normalized variance is plotted for the same two elements of the soil impedance matrix. It is defined, for each $\omega \in \mathbb{R}$, by

$$
g_{i j}^{\mathrm{P}}=\frac{\mathrm{E}\left[\mathbf{Z}_{\mathrm{P} i j}(\omega) \overline{\mathbf{Z}_{\mathrm{P} i j}(\omega)}\right]}{\mathrm{E}\left[\mathbf{Z}_{\mathrm{P} i j}(\omega)\right]^{2}},
$$

where $\mathbf{Z}_{\mathrm{P} i j}(\omega)$ is the element $(i, j)$ of matrix $\left[\mathbf{Z}_{\mathbf{P}}(\omega)\right]$, and $\bar{a}$ indicates the conjugate of the complex number $a$.

Two main facts can be observed on figure 3:

$-g_{i j}^{\mathrm{P}}(\omega)$ generally increases with frequency and in the neighborhood of resonance frequencies (here, the first compressional - for pumping - and shear - for shaking resonances of the layer of soil at $5 \mathrm{~Hz}$ and $2.5 \mathrm{~Hz}$, respectively).

$-g_{i j}^{\mathrm{P}}(\omega)$ is much larger for the pumping element than for the shaking element. 


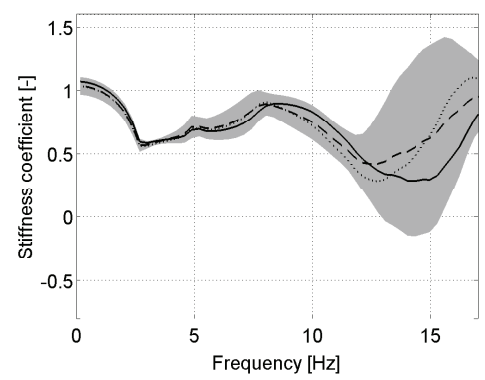

(a) Real part of shaking element

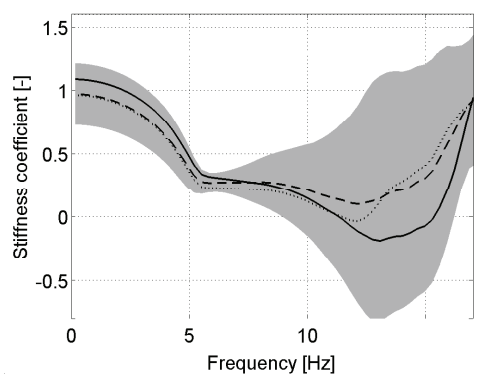

(b) Real part of pumping element

Figure 2. Real part of (a) the shaking and (b) the pumping elements of $\left[\mathbf{Z}_{P}(\omega)\right]$ : mean model (dotted line), one Monte Carlo trial (solid line), mean value (dashed line), and 90\%-confidence interval (shaded area).

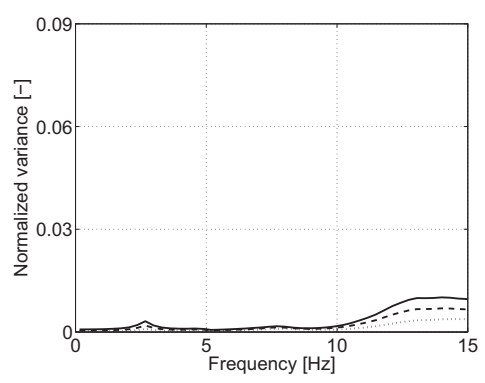

(a) Shaking element

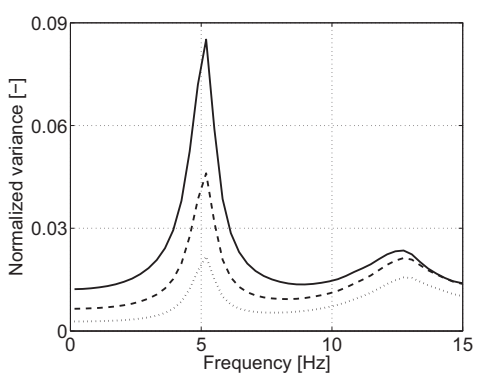

(b) Pumping element

Figure 3. Normalized variance $g_{i j}^{P}$ of the (a) shaking and (b) pumping elements of $\left[\mathbf{Z}_{P}(\omega)\right]$, for three lengths of correlation: $L_{c}=5 \mathrm{~m}$ (dotted line), $L_{c}=10 \mathrm{~m}$ (dashed line), and $L_{c}=20 \mathrm{~m}$ (solid line)

The first item is also observed on the other elements of the impedance matrix, that are not plotted here for concision. The second item is also observed, but to a lesser extent, when comparing the rocking and torsional elements, which correspond respectively to a compression and a shear deformation of the layer of soil.

The difference in the amplitudes of $g_{i j}^{\mathrm{P}}$ for the shaking and pumping elements of $\left[\mathbf{Z}_{\mathrm{P}}(\omega)\right]$ arise from the error that is made by replacing the reference problem by the SFE model. By doing this, the volume of soil that surrounds the sides of the embedded foundation, which is heterogeneous in the reference problem, is replaced by a homogeneous volume of soil. This has little impact on the random model of the 


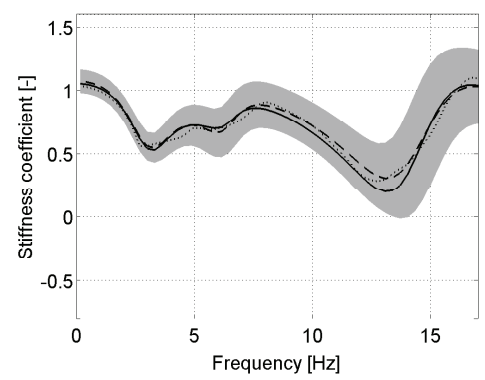

(a) Real part of shaking element

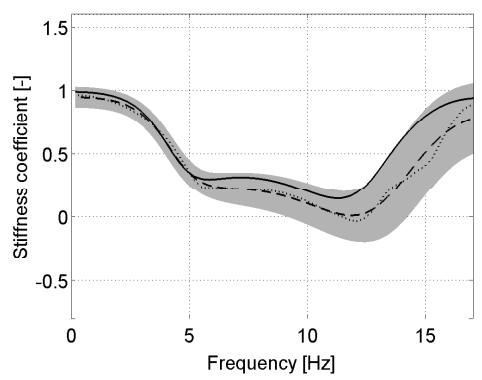

(b) Real part of pumping element

Figure 4. Real part of (a) the shaking and (b) the pumping elements of $\left[\mathbf{Z}_{N P}(\omega)\right]$ : mean model (dotted line), one Monte Carlo trial (solid line), mean value (dashed line), and 90\%-confidence interval (shaded area).

pumping element of $\left[\mathbf{Z}_{\mathrm{P}}(\omega)\right]$, because the dynamics of the vertical displacements of the foundation are more influenced by the volume of soil beneath the foundation. On the horizontal displacements, however, this introduces a very important model error, virtually canceling any variability on the shaking element of $\left[\mathbf{Z}_{\mathrm{P}}(\omega)\right]$.

\subsection{Nonparametric model of the impedance matrix}

We then turn to the construction of the nonparametric model of the soil impedance matrix $\left[\mathbf{Z}_{\mathrm{NP}}(\omega)\right]$. It is based on the mean model of the reference problem (figure 1(b)), where all mechanical parameters are considered constant within the layer of soil, at their mean values (see above). Besides the mean model, the dispersion parameters $\delta_{\mathrm{K}}$, $\delta_{\mathrm{D}}$, and $\delta_{\mathrm{M}}$ also have to be selected. We choose here, for illustration, $\delta_{\mathrm{K}}=\delta_{\mathrm{D}}=$ $\delta_{\mathrm{M}}=0.1$, and will discuss this choice further in the next section.

The real parts of the nonparametric model of the shaking and pumping elements of the soil impedance matrix are drawn in figure 4. The corresponding normalized variances $g_{i j}^{\mathrm{NP}}$ (eq. [9]) are plotted on figure 5. Two facts are noticeable:

- Although the amplitude is different, the dynamical shape of the normalized variances for $\left[\mathbf{Z}_{\mathrm{NP}}(\omega)\right]$ is similar to that of $\left[\mathbf{Z}_{\mathrm{P}}(\omega)\right]$.

- The shaking and pumping elements of $\left[\mathbf{Z}_{\mathrm{NP}}(\omega)\right]$ yield similar levels of variability, while the levels were very different in the parametric case. 


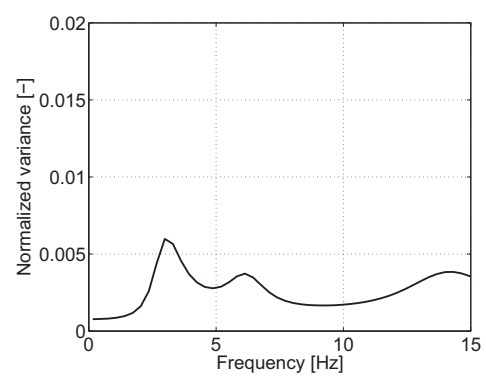

(a) Shaking element

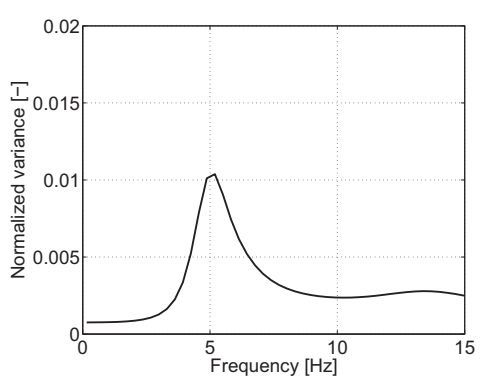

(b) Pumping element

Figure 5. Normalized variance $g_{i j}^{N P}$ of the (a) shaking and (b) pumping elements of $\left[\mathbf{Z}_{N P}(\omega)\right]$, for $\delta_{\mathrm{K}}=\delta_{\mathrm{D}}=\delta_{\mathrm{M}}=0.1$.

\subsection{Discussion}

As already noted, in the parametric approach, a model error is introduced by considering heterogeneous Lamé's parameters fields only in a bounded volume of soil under the foundation, rather than everywhere in the layer, as in the reference problem. This error has influence on the shaking element of $\left[\mathbf{Z}_{\mathrm{P}}(\omega)\right]$, but little on the pumping element, due to the location of the heterogeneous volume. In the nonparametric approach, there is no such difference between the elements as model errors are taken into on all terms of the impedance matrix.

It is therefore reasonable to assess that the pumping elements of both models of the impedance matrix represent comparable physical problems, while this is not the case for the shaking elements. It is then proposed to identify the dispersion parameters $\delta_{\mathrm{K}}$, $\delta_{\mathrm{D}}$, and $\delta_{\mathrm{M}}$ on the values of the correlation coefficients of the pumping element, or such that the following cost function is minimized

$$
\epsilon=\left\|g_{\text {pump }}^{\mathrm{P}}-g_{\text {pump }}^{\mathrm{NP}}\right\|^{2},
$$

where the notations used are obvious generalizations of eq. [9]. Other identification methods, more appropriate for uncertainty quantification, are currently investigated, and will be discussed elsewhere.

For simplicity, we present only results of the identification of the dispersion parameters with the additional simplification that $\delta_{\mathrm{K}}=\delta_{\mathrm{D}}=\delta_{\mathrm{M}}$. In that case, the inverse problem can be solved simply by sampling the parameter space (1-dimensional here) and solving one problem for each sample. A more complete identification process would require the independent identification of each dispersion parameter, as well as the concomitant identification of the mean model of the impedance matrix $\left[\mathrm{Z}_{0}(\omega)\right]$. In that case, more complex sampling procedures will be necessary. The results obtained for the three $L_{c}$ that were considered are presented in figure 6 . 


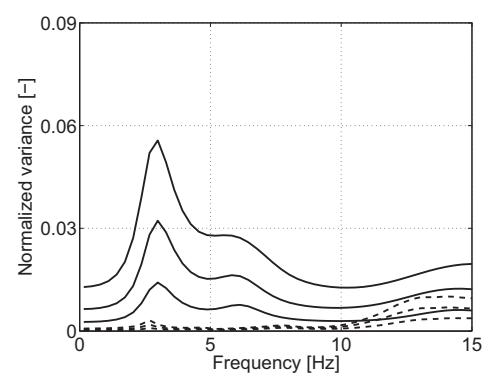

(a) Shaking element

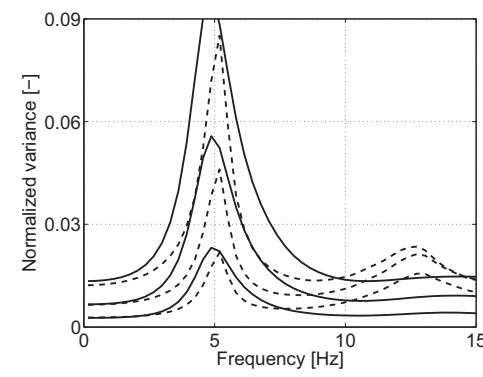

(b) Pumping element

Figure 6. Normalized variances for $\left[\mathbf{Z}_{P}(\omega)\right]$ (dashed lines), as in figure 3, for $L_{c}=5$ $m$ (lowest variability), $L_{c}=10 \mathrm{~m}$, and $L_{c}=20 \mathrm{~m}$, and corresponding correlation coefficients for $\left[\mathbf{Z}_{N P}(\omega)\right]$ (solid lines), with $\delta_{\mathrm{K}}=\delta_{\mathrm{D}}=\delta_{\mathrm{M}}=0.15$ (lowest variability), $\delta_{\mathrm{K}}=\delta_{\mathrm{D}}=\delta_{\mathrm{M}}=0.2$, and $\delta_{\mathrm{K}}=\delta_{\mathrm{D}}=\delta_{\mathrm{M}}=0.3$.

\section{Conclusion}

Two approaches were presented in this paper for the modeling of a reference random problem. The parametric approach, appropriate for the modeling of data uncertainties, was shown to introduce a model error by the discretization of the random mechanical parameters fields. However, on the example of the foundation embedded in a layer of random soil on a rigid bedrock, this model error seems to have little effect on the shaking element of the impedance matrix, and more on the pumping element.

The nonparametric approach, accounts for both model errors and data uncertainties. It is based on the mean model of the impedance matrix, in which a large model error is introduced by comparison with the reference model. On the example presented here, it was shown to yield more balanced levels of variability for the shaking and pumping elements of the impedance matrix than for the parametric approach. Finally, a novel method was proposed for the identification of the dispersion parameters of the nonparametric method, based on results for the pumping element of the impedance matrix with the parametric approach.

In a forthcoming paper, we will present results using more appropriate identification schemes, based in particular on the works of (Arnst, 2007; Arnst et al., 2007; Soize, 2005), and with the simultaneous identification of the dispersion parameters and of the mean model of the impedance matrix.

\section{References}

Arnst M., Inversion of probabilistic models of structures using measured transfer functions, PhD thesis, École Centrale Paris, Châtenay-Malabry, France, April, 2007. 
$121^{\text {re }}$ soumission à European Journal of Computational Mechanics

Arnst M., Clouteau D., Bonnet M., "Identification of non-parametric probabilistic models from measured frequency transfer functions", Computer Methods in Applied Mechanics and Engineering, 2007. Submitted in revised form.

Cottereau R., Probabilistic models of impedance matrices. Application to dynamic soil-structure interaction, PhD thesis, École Centrale Paris, Châtenay-Malabry, France, January, 2007.

Cottereau R., Clouteau D., Soize C., " Construction of a probabilistic model for impedance matrices", Computer Methods in Applied Mechanics and Engineering, vol. 196, n 17-20, p. 2252-2268, March, 2007a.

Cottereau R., Clouteau D., Soize C., "Probabilistic impedance of foundation: impact on the seismic design on uncertain soils", Earthquake Engineering and Structural Dynamics, 2007b. Submitted.

Ghanem R. G., Spanos P. D., Stochastic Finite Elements: A Spectral Approach, Springer-Verlag, 1991.

Magoulès F., Harari I., “ Absorbing boundary conditions”, Computer Methods in Applied Mechanics and Engineering, vol. 195, n²9-32, p. 3551-3902, June, 2006.

Savin É., Clouteau D., “ Elastic wave propagation in a 3D unbounded random heterogeneous medium coupled with a bounded medium. Applications to seismic soil-structure interaction.", International Journal for Numerical Methods in Engineering, vol. 54, n 4, p. $607-$ 630, 2002.

Soize C., " A nonparametric model of random uncertainties for reduced matrix models in structural dynamics”, Probabilistic Engineering Mechanics, vol. 15, p. 277-294, 2000.

Soize C., " Maximum entropy approach for modeling random uncertainties in transient elastodynamics", Journal of the Acoustical Society of America, vol. 109, n 5, p. 1979-1996, May, 2001.

Soize C., " Random matrix theory for modeling uncertainties in computational mechanics", Computer Methods in Applied Mechanics and Engineering, vol. 194, p. 1333-1366, 2005. 